\title{
Joint Source-Channel Coding and Guessing ${ }^{1}$
}

\author{
Erdal Arikan and Neri Merhav \\ Dept. of Electrical and Electronics Eng., Bilkent University, 06533 Ankara, Turkey. arikan eee.bilkent.edu.tr \\ Dept. of Electrical Eng., Technion - I.I.T., Haifa 32000, Israel. merhaveee.technion.ac.il
}

\begin{abstract}
We consider the joint source-channel guessing problem, define measures of optimum performance, and give single-letter characterizations. As an application, sequential decoding is considered.
\end{abstract}

\section{Introduction and Main Result}

Let $P$ be a discrete memoryless source over a finite alphabet $\mathcal{U}, \hat{U}$ a reconstruction alphabet, and $d$ a single-letter distortion measure defined on $\mathcal{U} \times \hat{\mathcal{U}}$. A $D$-admissible guessing strategy for $\mathcal{U}^{N}$ is a possibly infinite ordered list $\mathcal{G}_{N}=\left\{\hat{\mathbf{u}}_{1}, \hat{\mathbf{u}}_{2}, \ldots\right\} \subset$ $\hat{\mathcal{U}}^{N}$ such that for each $\mathbf{u} \in \mathcal{U}^{N}$ there exists $\hat{\mathbf{u}}_{i} \in \mathcal{G}_{N}$ with $d\left(\mathbf{u}, \hat{\mathbf{u}}_{i}\right) \leq N D$. The guessing function $G_{N}(\cdot)$ induced by a guessing strategy $\mathcal{G}_{N}$ is the function that maps each $\mathbf{u} \in \mathcal{U}^{N}$ into the index $i$ of the first $\hat{\mathbf{u}}_{i} \in \mathcal{G}_{N}$ such that $d\left(\mathbf{u}, \hat{\mathbf{u}}_{i}\right) \leq$ $N D$. Thus, $G_{N}(\mathbf{u})$ is the number of guesses required to find a reconstruction of $\mathbf{u}$ within distortion level $N D$ by sequentially probing from the list $\mathcal{G}_{N}$. The moments $\mathbf{E}\left[G_{N}(\mathbf{U})^{\rho}\right], \rho \geq 0$, serve as measures of complexity for the guessing effort. Arikan and Merhav [1] defined the guessing exponent as

$$
E(D, \rho)=\lim _{N \rightarrow \infty} \frac{1}{N} \inf _{\mathcal{G}_{N}} \ln \mathbf{E}\left[G_{N}(\mathrm{U})^{\rho}\right]
$$

for $\rho \geq 0$, and showed that it has a single-letter form given by

$$
E(D, \rho)=\max _{Q}[\rho R(D, Q)-D(Q \| P)]
$$

where $R(D, Q)$ is the rate-distortion function, $D(Q \| P)$ is the relative entropy, and the maximum is over all probability distributions on $\mathcal{U}$.

The aim of this talk is to consider the guessing problem in a joint source-channel setting, in which one is allowed to send information about $U$ to the guesser over some discrete memoryless channel $W$, using the channel $\lambda$ times for each source symbol. We assume $W$ has a finite input alphabet $\mathcal{X}$ and a finite output alphabet $\mathcal{Y}$. The source output $\mathrm{U} \in \mathcal{U}^{N}$ is encoded into a channel input block $\mathrm{X} \in \mathcal{X}^{K}, K=\lceil\lambda N\rceil$, using an encoder $e_{N}: \mathcal{U}^{N} \rightarrow \mathcal{X}^{K}, \mathrm{X}$ is transmitted over $W$, and the guesser observes the channel output $Y \in \mathcal{Y}^{K}$. A $D$ admissible guessing scheme, in this situation, is a collection $\left\{\mathcal{G}_{N}(\mathbf{y}), \mathbf{y} \in \mathcal{Y}^{K}\right\}$, such that for each $\mathrm{y} \in \mathcal{Y}^{K}, \mathcal{G}_{N}(\mathbf{y}) \subset \hat{\mathcal{U}}^{N}$ is a $D$-admissible guessing scheme for $U^{N}$ in the previously defined sense. We define the joint source-channel guessing exponent as

$$
E_{s c}(D, \rho)=\lim _{N \rightarrow \infty} \frac{1}{N} \inf _{e_{N}, \mathcal{G}_{N}} \ln \mathbf{E}\left[G_{N}(\mathbf{U} \mid \mathbf{Y})^{\rho}\right]
$$

Here, $G_{N}(\mathbf{U} \mid \mathbf{Y})$ denotes the guessing function induced by $\mathcal{G}_{N}(\mathbf{Y})$. Our main result is the following.

\footnotetext{
${ }^{1}$ The work of N. Merhav was partially supported by the Wolfson Research Awards administered by the Israel Academy of Sciences and Humanities.
}

Theorem 1 The joint source-channel guessing exponent is given by

$$
E_{s c}(D, \rho)=\max \left\{0, E(D, \rho)-\lambda E_{0}(\rho)\right\}
$$

where $E_{0}(\rho)$ is Gallager's function [2, p.138] for $W$.

\section{LIST-ERRor EXPONENT}

Consider list-decoding in the above situation so that given the channel output $\mathbf{Y}$ one is allowed to generate $\ell$ estimates of the source output $U$ and suppose an error occurs only if none of the estimates is within distortion level $N D$ of $\mathrm{U}$. Let $P_{e, N}$ denote the minimum possible value of the list decoding error probability over all encoders $e_{N}$ and all list- $\ell$ decoders. The asymptotic behavior of $P_{e, N}$ for $\ell=1$ has been considered by Csiszár, but it remains only partially known. Here, we consider exponential list sizes, $\ell=e^{N L}$, and define the joint source-channel list-error exponent as

$$
F_{s c}(L, D)=\lim _{N \rightarrow \infty}-\frac{1}{N} \log P_{e, N}
$$

Our second result is the following.

Theorem 2 The joint source-channel list-error exponent is given by

$$
F_{s c}(L, D)=\inf _{R \geq L}\left[F(R, D)+\lambda E_{s p}[(R-L) / \lambda]\right]
$$

where $F(R, D)$ is Marton's source coding exponent [4] and $E_{s p}(\cdot)$ is the sphere-packing exponent [2, p. 157].

\section{Application to Sequential Decoding} Koshelev [5] considered using sequential decoding in joint source-channel coding systems for the lossless case $D=0$. Here, we prove the following converse which complements his result, and applies to the lossy case $D>0$ as well.

Theorem 3 For any $\rho \geq 0$, the $\rho$ th moment of computation in sequential decoding in a joint source-channel coding system must grow exponentially with the number of symbols correctly decoded if $E(D, \rho)>\lambda E_{0}(\rho)$.

\section{REFERENCES}

[1] E. Arikan and N. Merhav, "Guessing subject to distortion," Technion -I.I.T., EE Pub. No. 1015, Feb. 1996 (Also, submitted to IEEE Trans. Inform. Theory).

[2] R. G. Gallager, Information Theory and Reliable Transmission. New York: Wiley, 1968.

[3] I. Csiszár, "On the error exponent of source channel transmission with a distortion threshold," IEEE Trans. Inform. Theory, vol. IT-28, no. 6, pp. 823-828, Nov. 1982.

[4] K. Marton, "Error exponent for source coding with a fidelity criterion," IEEE Trans. Inform. Theory, vol. IT-20, pp. 197$199,1974$.

[5] V. N. Koshelev, "Direct sequential encoding and decoding for discrete sources," IEEE Trans. Inform. Theory, vol. IT-19, pp. 340-343, May 1973. 